Document downloaded from:

http://hdl.handle.net/10251/74626

This paper must be cited as:

Weber, B.; Pinggera, J.; Torres Bosch, MV.; Reichert, M. (2013). Change Patterns in Use: A Critical Evaluation. En Enterprise, Business-Process and Information Systems Modeling, BPMDS 2013. Springer Verlag. 261-276. doi:11007/978-3-642-38484-4_19.

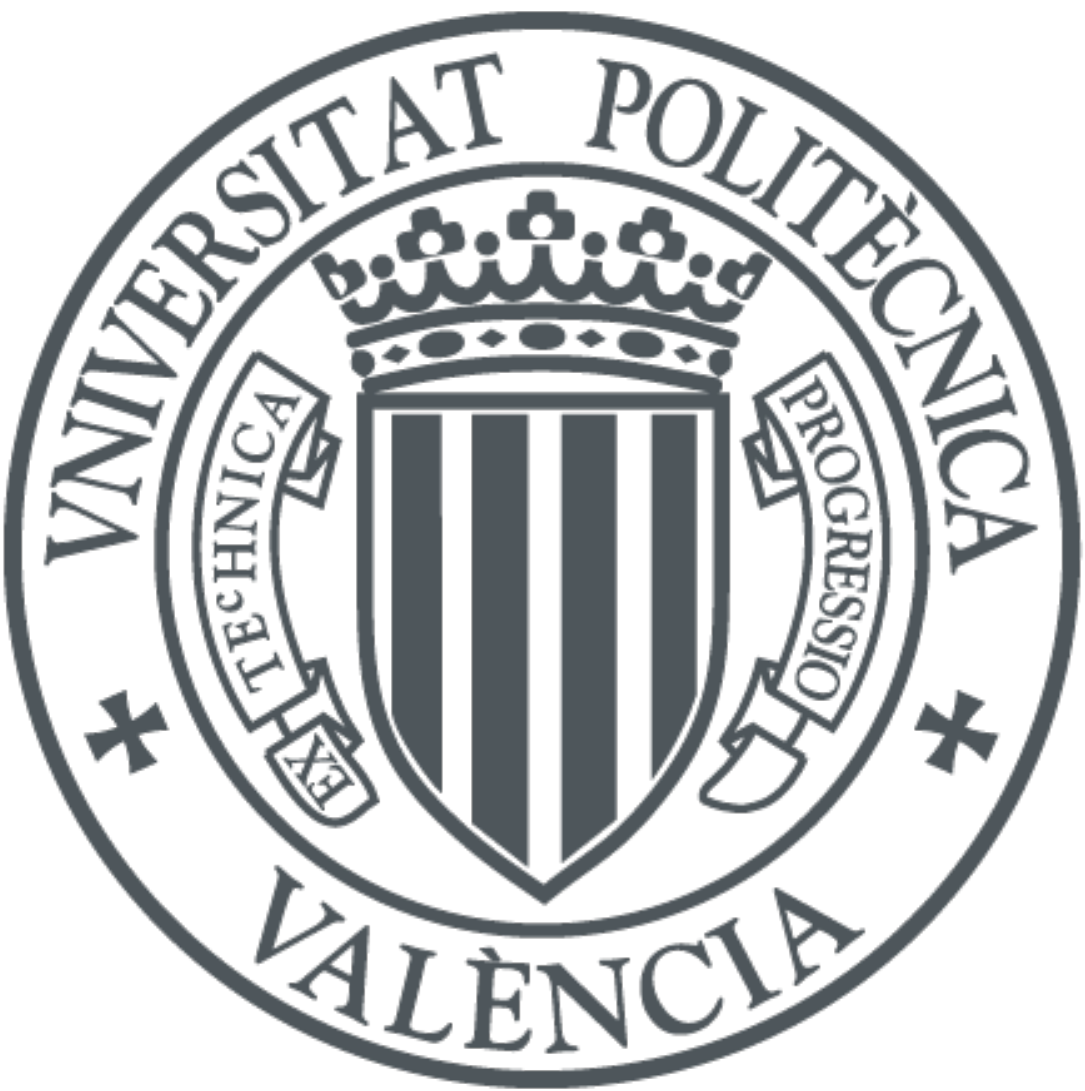

The final publication is available at

http://dx.doi.org/10.1007/978-3-642-38484-4_19

Copyright Springer Verlag

Additional Information 


\title{
Change Patterns in Use: A Critical Evaluation*
}

\author{
Barbara Weber $^{1}$, Jakob Pinggera ${ }^{1}$, Victoria Torres $^{2}$, and Manfred Reichert ${ }^{3}$ \\ 1 University of Innsbruck, Austria \\ \{barbara.weber, jakob. pinggera\}@uibk.ac.at \\ 2 Universitat Politècnica de València, Spain \\ vtorres@pros.upv.es \\ 3 University of Ulm, Germany \\ manfred.reichert@uni-ulm.de
}

\begin{abstract}
Process model quality has been an area of considerable research efforts. In this context, the correctness-by-construction principle of change patterns provides promising perspectives. However, using change patterns for model creation imposes a more structured way of modeling. While the process of process modeling (PPM) based on change primitives has been investigated, little is known about this process based on change patterns. To obtain a better understanding of the PPM when using change patterns, the arising challenges, and the subjective perceptions of process designers, we conduct an exploratory study. The results indicate that process designers face little problems as long as controlflow is simple, but have considerable problems with the usage of change patterns when complex, nested models have to be created. Finally, we outline how effective tool support for change patterns should be realized.
\end{abstract}

Key words: Process Model Quality, Process of Process Modeling, Change Patterns, Exploratory Study, Problem Solving

\section{Introduction}

Much conceptual, analytical, and empirical research has been conducted during the last decades to enhance our understanding of conceptual modeling. In particular, process models have gained significant importance due to their fundamental role for process-aware information systems [1]. Even though it is well known that a good understanding of a process model has a direct and measurable impact on the success of any modeling initiative [2], process models display a wide range of quality problems impeding their comprehensibility and hampering their maintainability $[3,4]$. Literature reports, for example, on error rates between $10 \%$ and $20 \%$ in industrial process model collections [3].

To improve process model quality, change patterns offer a promising perspective [4]. They have well-defined semantics [5] and combine change primitives (e.g., to add nodes or edges) to high-level change operations. Particularly appealing is correctness-by-construction [6, 7], i.e., the modeling environment only provides those change patterns to the process designers, which ensure that a sound process model is transformed into another sound process model.

\footnotetext{
* This research is supported by Austrian Science Fund (FWF): P23699-N23
} 
The use of change patterns implies a different way of creating process models, since correctness-by-construction imposes a structured way of modeling by enforcing block structuredness. Irrespective of whether change patterns or change primitives are used, model creation requires process designers to construct a mental model (i.e., internal representation) of the requirements to be captured in the process model [8]. In a subsequent step, the mental model is mapped to the constructs provided by the modeling language creating an external representation of the domain [8]. While the construction of the mental model presumably remains unaffected, the use of change patterns leads to different challenges concerning pattern selection and combination for creating the external representation. Further, the exact set of change patterns available influences the selection. While a large set of change patterns allows for more flexibility, it also increases complexity, making the modeling environment more difficult to use. Consequently, process designers might have to look several steps ahead to construct a certain process fragment, which constitutes a major difference compared to the use of change primitives, which do not impose any structural restrictions (i.e., no order is imposed when placing elements on the modeling canvas).

The process of creating process models based on change primitives has caused significant attention leading to a stream of research on the process of process modeling $(\mathrm{PPM})[8,9,10,11]$. This research is characterized by its focus on the formalization phase of model creation, i.e., the designer's interactions with the modeling environment [9]. Still, little is known about the PPM when utilizing change patterns. In this paper, we try to obtain an in-depth understanding of the PPM when using change patterns. In particular, this paper aims at understanding whether the necessity to look ahead leads to additional barriers during model creation and at shedding light on the challenges process designers face when using change patterns, which is essential to provide effective tool support.

To obtain an in-depth understanding of these issues, we implement a change pattern modeling editor based on Cheetah Experimental Platform (CEP) and conduct an exploratory study comprising several modeling tasks. The results of our study underline the potential for model creation based on change patterns. In particular, process designers did not face any major problem when using change patterns for constructing simple process fragments. When more complex process fragments forced process designers to look ahead, difficulties increased observably. Insights obtained from this exploratory study provide a better understanding of the PPM when using changes patterns and reveal challenges arising in such a context. In particular, results provide a contribution toward a better understanding on how tool features like change patterns impact the PPM, but also give advice on how effective tool support should be designed.

Sect. 2 introduces backgrounds. Sect. 3 describes the design of the exploratory study. Sect. 4 discusses challenges in change pattern use and Sect. 5 presents results on process designers' perception of patterns use. Results are discussed in Sect. 6. Related work is presented in Sect. 7. Sect. 8 concludes the paper. 


\section{Backgrounds}

This section briefly introduces change patterns and then presents cognitive foundations and backgrounds of the PPM.

\subsection{Process Modeling Based on Change Patterns}

Change patterns provide a different way of interacting with the modeling environment in modeling phases. Instead of specifying a set of change primitives, the process designer applies change patterns (i.e., high-level change operations) to realize the desired model adaptation (cf. Fig. 1). Examples of change patterns include the insertion and deletion of process fragments, or their embedding in loops (the whole catalogue can be found in [23, 4] and their semantics in [5]). When applying a single change primitive, soundness of the resulting process model cannot be guaranteed, but must be explicitly checked after applying the change primitives. On the contrary, change pattern implementations often associate pre-/post-conditions with high-level change operations to guarantee model correctness after each adaptation [6, 7]. Process editors applying the correctnessby-construction principle (e.g., [23]) usually provide only those change patterns to the process designer that allow transforming a sound process model into another sound one. This is realized by imposing structural restrictions on process models (e.g., block structuredness).

A) <Insert Node XOR-Split, Insert Control Edge, Insert Node XOR-Join>

B) <Embed Process Fragment in Conditional Branch>

Fig. 1. Set of Change Primitives (A) and Patterns (B) to make an activity optional

\subsection{Cognitive Foundations of Problem Solving}

We consider the creation of process models to be a complex problem solving task. Problem solving has been an area of vivid research in cognitive psychology for decades. Therefore, we turn to cognitive psychology to understand the processes followed by humans when solving a problem like creating a process model.

Schemata. A central insight from cognitive research is that the human brain contains specialized regions contributing different functionality to the process of solving complex problems. Long-term memory is responsible for permanently storing information and has an essentially unlimited capacity, while in working memory comparing, computing and reasoning take place [12]. Although the latter is the main working area of the brain, it can store only a limited amount of information, which is forgotten after 20-30 seconds if not refreshed [13]. The question arises how information can be processed with such limited capacity. The human mind organizes information in interconnected schemata rather than in isolation [12]. Those schemata, stored in long-term memory, incorporate general concepts of similar situations [12]. Whenever situations similar to a schema arise, the latter is retrieved to help organizing information by creating chunks of 
information that can be processed efficiently [14]. For instance, when asked to create a process model using change patterns, a designer must create an internal representation of the problem [8]. For this, information about the domain is retrieved and organized in memory using existing schemata for process modeling. Therefore, schemata guide the comprehension process, helping to re-organize information for its processing in working memory [14].

Problem-Solving Strategies. When confronting novices with an unfamiliar problem they cannot rely on specialized problem solving strategies. Instead, they must find a way to solve the problem and come up with an initial skeletal plan [15]. Then, novices utilize general problem solving strategies, like meansends analysis, due to the lack of more specific strategies for the task at hand [16]. Means-ends analysis can be described as the continual comparison of the problem's current state with the desired end product. Based on this, the next steps are selected until a satisfying solution is found [16]. After applying the constructed plan, it can be stored in long-term memory as plan schema [15]. For this, task-specific details are removed from the plan schema resulting in a plan schema that can be automatically applied in similar situations [17]. When confronted with a problem solving task in the future, the appropriate plan schema is selected using a case-based reasoning approach [18]. The retrieved plan schema provides the user with structured knowledge that drives the process of solving the problem [14, 18]. Plan schemata allow experts to immediately decide what steps to apply to end up with the desired solution [19]. If the plan schema is well developed, an expert never reaches a dead end when solving the problem [20].

Plan schemata seem to be important when creating process models based on change patterns since change pattern cannot be combined in an arbitrary manner. If no plan schema is available on how to combine change patterns to create the desired process model, designers have to utilize means-ends analysis until a satisfying solution is found. This behavior is more likely to reach in detours, reducing the process designer's efficiency when creating process models.

\subsection{The Process of Process Modeling}

During the formalization phase, process designers create a syntactically correct process model reflecting a given domain description by interacting with the process editor [21]. The formalization, i.e., the PPM, can be described as a cycle of the three phases of comprehension, modeling and reconciliation $[9,8]$.

Comprehension. In comprehension phases designers try to understand the requirements to be modeled by extracting information from the task description and the existing process model to build an internal representation of the problem in working memory $[16,20]$. Depending on the availability of schemata for organizing the acquired knowledge, working memory is utilized more or less efficiently. If the process designer has solved a similar problem previously (i.e., a plan schema for the problem is stored in long-term memory), he can directly create the process model without any further attention on which steps to execute or plan next. Either way, working memory is filled with knowledge extracted from requirements and, if available, the process model being created. 
Modeling. The designer uses the internal representation developed during the comprehension phase to materialize the solution in a process model (by creating or changing it) $[8,9]$. Hence, modeling phases consist of a set of structural model adaptations. More specifically, designers interact with the modeling environment using change primitives such as add activity or add edge. The materialization of a process model adaptation may require the joint application of several change primitives. For instance, making an activity optional may require the application of three change primitives as shown in Fig. 1A. Once all information stored in working memory is incorporated in the process model, the designer interrupts the modeling endeavor to incorporate additional requirements into the internal representation [9].

Reconciliation. Designers may reorganize a process model (e.g., renaming of activities) and utilize its secondary notation (e.g., notation of layout, typographic cues) to enhance understandability [22]. However, the number of reconciliation phases in the PPM depends on the designer's ability of placing elements correctly when creating them [9].

\section{Exploratory Study}

To gain a better understanding of the PPM using change patterns we conduct an exploratory study. This section describes research questions and study design.

Research Questions. Central aim is to obtain an in-depth understanding of the PPM when using change patterns. More specifically, we try to understand the challenges designers are facing when using change patterns for model creation. Respective challenges can result in detours for designers on their way to a complete process model. Detours during process modeling result in decreased problem solving efficiency. Moreover, they might lead to modeling errors that persist in the final model.

RQ1: What are re-occurring challenges in the usage of change patterns that designers face and where do these challenges originate from?

The study also aims at investigating how designers experience their interaction with the modeling environment to create process models using change pattern.

RQ2: What is the subjective perception of designers when using change patterns for model creation?

Exploratory Study Execution. The design of the study consists of three phases. In the first phase, demographic data is collected. In the second phase, two modeling tasks are executed. When working on the modeling tasks all interactions with the modeling environment are recorded using CEP [24]. This allows us to replay the creation of the process model step-by-step (cf. [24, 9]), addressing RQ1. After completing the modeling tasks, Perceived Ease of Use and the Perceived Usefulness of Technology Acceptance Model (TAM) [25] are assessed to investigate RQ2. In addition, participating subjects are asked to provide feedback regarding their experiences. 
Subjects. The exploratory study was conducted in Innsbruck with 16 students of a graduate course on business process management. Since designers in practical settings are often not expert designers, but rather casual designers that only obtained a basic amount of training [26], we did not require modeling experts for our study. Subjects obtained basic training in modeling business processes prior to the exploratory study. In addition, they were taught theoretical backgrounds on change patterns prior to the exploratory study, but did not have any hands-on experience in the creation of process models using change patterns.

Modeling Tasks. For Task A, subjects received an informal requirements description and the solution of the modeling task (i.e., a process model). Subjects had to re-model the process using change patterns. Since subjects had the correct solution available, the challenge lies in determining the patterns to use for re-building the model and how to combine them effectively. This allowed students to develop problem solving strategies for utilizing change pattern. Task A was a process run by the "Task Force Earthquakes" of the German Research Center for Geosciences [27]. Subjects were asked to model the "Transport of Equipment" process using change patterns. The task requires sequences as well as conditional/parallel branchings. The solution model has a nesting depth of 2 .

In Task B, designers had to create a process model starting from an informal description. This time, no solution model was made available to the subjects. Consequently, they not only had to decide which patterns to use and how to combine them, but additionally had to develop an understanding of the domain (by creating a mental model) and map it to the available change patterns. Therefore, schemata for extracting information from the textual description were necessary for completing the modeling task (e.g., for identifying activities). Task $\mathrm{B}$ describes the pre-take off procedures for a general aviation flight under visual rules [28]. Like Task A, it comprises sequences and conditional/parallel branchings. In terms of complexity, it only has a nesting depth of 1 .

Change Pattern Set. When devising a modeling environment for model creation based on change patterns the question arises which change pattern set to provide. While a large set offers more flexibility, it also increases complexityespecially when mapping the mental model to the available patterns. We therefore utilize a minimal change pattern set (for the full pattern set see [4]), which allows designers to create all main control-flow constructs for tasks A and B (i.e., sequences, parallel branches, conditional branchings, and loops). The following patterns were available to the designers: AP1 (Insert Process Fragment), AP2 (Delete Process Fragment), AP8 (Embed Fragment in Loop), AP10 (Embed Process Fragment in Conditional Branch), and AP13 (Update Condition). Concerning AP1, two pattern variants were provided: Serial Insert and Parallel Insert.

\section{Challenges in the Usage of Change Patterns}

This section describes the data analysis procedure applied to obtain an in-depth understanding of re-occurring challenges during the PPM based on change patterns, answering RQ1 it further presents obtained results for Tasks A and B. 


\subsection{Data Analysis Procedure}

Step 1: Determine Solution Model, Distance, and Optimal Problem Solving Paths. In a first step, for each modeling task, we create a model representing the correct solution (i.e., $S_{S}$ ). In a next step, we establish the distance for transforming an empty model (i.e., $\left.S_{0}\right)$ to $S_{S}$ (i.e., the minimum number of change patterns needed). Typically, the process designer has several possibilities to create a solution model $S_{S}$ by starting from $S_{0}$ and applying a sequence of model transformations. From a cognitive perspective, each possible sequence of change patterns that leads without detours to the correct solution constitutes an optimal problem solving path. For example, inserting region R4 of Task B in Fig. 2B starting from an empty modeling canvas can be achieved in different ways with a minimum number of 4 change patterns (e.g., $S^{\prime}$ can be created by first inserting $R 4.1$, next embedding $R_{4} .1$ in a conditional branch, then inserting $R 4.2$, and finally updating the transition condition).

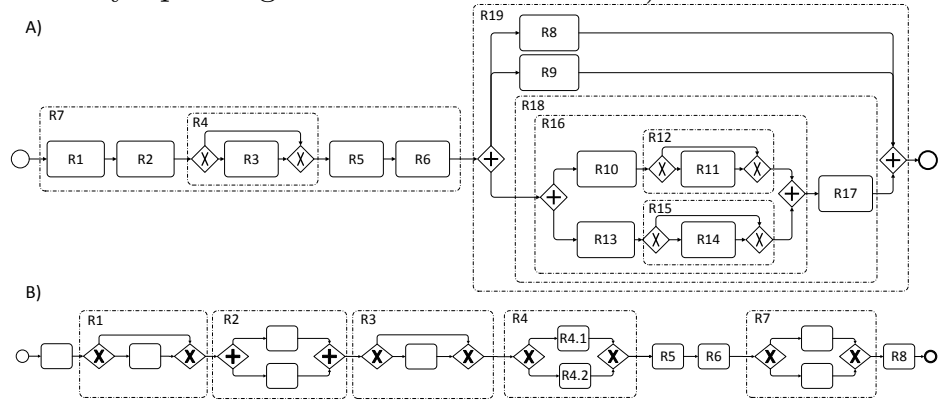

Fig. 2. Solution Models for Tasks A and B

Step 2: Determine Deviations from Optimal Problem Solving Path. To identify potential challenges designers were facing we analyze their problem solving paths for both modeling tasks using the replay functionality of CEP. For this, for each process designer we compare the problem solving path $P_{0, S}$ (i.e., sequence of patterns to transform $S_{0}$ to $S_{S}$ ) and capture deviations from the optimal problem solving path. Respective deviations can be detours the designer takes until coming up with the correct solution. Deviations quantify how efficient the chosen problem solving strategy is - denoted as process deviations. However, deviations can also be discrepancies between the model created by designers and the solution model $S_{S}$, denoted as product deviations. Fig. 3 shows the problem solving path of one process designer, who managed to model region R4 of Task B in Fig. $2 \mathrm{~B}$ correctly (i.e., 0 product deviations), but made a detour of 2 change patterns (crossed out lines) before reaching the solution (i.e., the solution path $P_{0, S}$ comprises 2 superfluous change patterns summing up to 2 process deviations).

Step 3: Classification of Deviations and Aggregation of Deviations. In Step 3, using the replay functionality of CEP, deviations are mapped to regions of the process model and reasons for every deviation are identified (e.g., misinterpretation of the textual description, problems with usage of patterns) in an iterative consensus-building process [29]. Moreover, to obtain an overview of which model parts caused most difficulties we aggregate for each task deviations per region. 


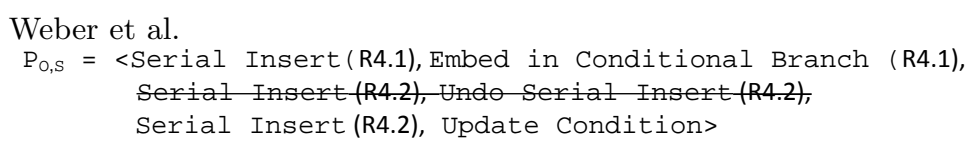

Fig. 3. Problem Solving Path with 2 Process Deviations

\subsection{Results Related to Task A}

Regarding Task A, a minimum of 18 operations is needed to create the correct solution model (cf. Fig. 2A). Overall, we identified 254 deviations - 232 process deviations (i.e., detours in the modeling process) and 22 product deviations (i.e., deviations of the final models from the solution model) (cf. Table 1). Process deviations per process designer ranged from 0 to 58 , with an average of 13.4 deviations. In turn, product deviations ranged from 0 to 8 per process designer, with an average of 1.3 deviations.

\begin{tabular}{|l|c|c|c|c|}
\hline Overview of deviations & \multicolumn{3}{|c|}{ Task A } & Task B \\
\hline & $\mathrm{R} 7$ & $\mathrm{R} 19$ & Overall & \\
\hline Overall deviations (Total) & 20 & 234 & 254 & 133 \\
\hline Process deviations (Total) & 18 & 214 & 232 & 88 \\
\hline Product deviations (Total) & 2 & 20 & 22 & 45 \\
\hline Overall deviations (Avg. / designer) & 1.3 & 14.6 & 15.9 & 8.3 \\
\hline Process deviations (Avg. / designer) & 1.1 & 13.4 & 14.5 & 5.5 \\
\hline Product deviations (Avg. / designer) & 0.1 & 1.3 & 1.4 & 2.8 \\
\hline Deviations per category & 13 & 61 & 74 & 36 \\
\hline Wrong pattern, wrong parameters & 4 & 79 & 83 & 10 \\
\hline Pattern testing/learning; trials & 0 & 0 & 0 & 30 \\
\hline Problems with pattern implementation & NA & NA & NA & 12 \\
\hline Problems with identification of activities & 0 & 57 & 57 & 0 \\
\hline Dead end and dead end resolution & 1 & 17 & 18 & 0 \\
\hline Other
\end{tabular}

Table 1. Overview of Results for Task A and Task B

Most of the designers started the modeling with little problems (i.e., from the 232 process deviations only 18 are related to region R7) that were mostly caused by wrong pattern usage (e.g., loop instead of conditional) or wrong parameter settings (e.g., activity inserted at wrong position). The remaining 214 deviations occurred in region R19, which only comprises two activities more than R7, but has higher structural complexity. Interestingly, when combining the patterns in an optimal way, the creation of both parts requires a similar number of change patterns (i.e., 7 patterns for R7 and 11 patterns for R19). When analyzing the process deviations related to R19, it turned out that process designers especially faced difficulties in creating region R16. Its nested structure forces designers to apply patterns in a certain ordering (i.e., requires an effective problem solving strategy including look ahead). For example, assuming that R8, R9, and R10 have already been inserted, the insertion of R13 in parallel to the already inserted regions leads to a dead end (i.e., the solution model can only be reached by deleting already created parts). Most designers (11 out of 16) ended up at least once in a dead end when trying to create the solution model. 3 of these 11 designers did not try to resolve the dead end, i.e., R16 is modeled incorrectly 
in their final models. The remaining designers (8 out of 11) tried to resolve the dead end by backtracking in the modeling process (i.e., 57 deviations). Partially, it took them several trials until they found a problem solving strategy suitable for constructing the respective fragment (i.e., 79 deviations). Strategies for constructing R16 included the usage of dummy activities and experiments to test and learn the functioning of the patterns. In turn, 5 out of 16 designers faced relatively little difficulties with the creation of this fragment since their initial strategy turned out to be effective, i.e., they were able to build the solution model in a straight-forward manner. In addition to problems related to the creation of R16, process deviations were caused in the context of single activities of region R19. Again, the usage of wrong patterns or wrong parameter values was the primary source of deviations (i.e., 61 deviations).

\subsection{Results Related to Task B}

Regarding Task B, a minimum of 19 change operations is needed to create the correct solution model. Overall, we identified 133 deviations, 88 process deviations and 45 product deviations (cf. Table 1). Process deviations per process designer ranged from 1 to 17 with an average of 5.5 deviations. Product deviations per designer ranged from 0 to 5 deviations with an average of 2.8 deviations. Product deviations were partially caused by ambiguities in the textual description, partially by mismatches between the textual description and the final models and presumably rather stem from problems with the domain than from actual pattern usage. Since subjects did not have a process model given as a template like in Task A, but had to build the model themselves starting from an informal requirements description, it is little surprising that the percentage of product deviations is much higher compared to Task A. Regarding process deviations, for 12 out of 88 , there is clear evidence that they stem from problems with the domain rather than from problems caused by pattern usage (i.e., subjects were not sure whether to include certain model parts as activities and presumably lacked schemata for information extraction). For 30 out of 88 process deviations, there are clear indications that they were caused by problems in pattern usage. As detailed later on, all these deviations occurred in the context of region R4 and are the result of tooling problems, i.e., caused by patterns implementation. Additional 10 deviations seem to stem from problems in pattern usage and were caused by process designers testing the functioning of certain patterns. In turn, the remaining 36 deviations were caused by process designers initially selecting the wrong patterns (e.g., embedding an activity in a loop instead of embedding it in a conditional branch), and by using the correct pattern with incorrect parameters (e.g., inserting the correct activity at a wrong position). Even though most of these deviations rather seem to be domain related, we cannot conclude with certainty from our data whether this indicates problems caused by pattern usage (i.e., mapping the mental model to the available patterns) or problems with the domain (i.e., incorrect mental model).

Overall, subjects faced relatively little problems related to the usage of patterns when working on Task B. In particular, they did not have any notable 
problem when inserting activities in a sequence, making an activity optional, or inserting an activity in parallel to another one. The only exception was a region with two exclusive branches, which caused significant problems (cf. R4 in Fig. 4). To model this region correctly, process designers had to first insert one of the activities using pattern Serial Insert, subsequently use pattern Embed Process Fragment in Conditional Branch to make the previously inserted activity optional, then use pattern Serial Insert to insert the second activity, and finally use pattern Update Condition to insert a transition condition. Even though the subjects started correctly in the construction of this region-problems with the automatic layout-CEP made 5 out of 16 process designers think that the second Serial Insert pattern has not been applied correctly resulting into partially long detours (cf. Fig. 4). As a consequence, they tried to apply the pattern several times even though their initial solution would have been correct. 4 of the 5 process designers facing this problem realized after a few trials that the pattern had been applied correctly. Just 1 of 5 process designers, however, created a workaround solution that correctly reflected the requirements, but was a bit more complicated than the optimal solution (i.e., instead of creating one process fragment with two conditional branches, this designer created two process fragments with one optional activity each). Overall 30 out of 88 process deviations were caused by this problem. Interestingly, when faced with the same modeling structure later in the modeling process again (i.e., R7), the process designers did not have these problems anymore, but apparently learned how to use the patterns in combination to model such construct (4 process designers) or how to circumvent the situation with a workaround (1 process designer). While process designers had no problem solving strategy available when constructing R4, they could rely on the plan schemata developed in R4 for the construction of R7.

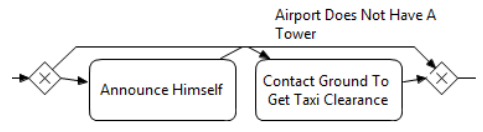

Fig. 4. Problem with Combined Pattern Usage

\section{Subjective Perception of Model Creation}

This section addresses research question $\mathrm{R} 2$ which deals with the subjective perception of process designers using change patterns. We investigate their ease of use and perceived usefulness for creating process models and discuss feedback provided by the participants after the exploratory study.

\subsection{Perceived Ease of Use and Perceived Usefulness}

To assess in how far process designers with moderate process modeling knowledge consider the CEP change pattern modeler as easy to use and useful, we asked them to fill out the Perceived Ease of Use and the Perceived Usefulness scales from the Technology Acceptance Model (TAM) [25] after the modeling session. 
Both scales consist of six 7-point Likert items, ranging from Extremely likely (1) over Neither Likely nor Unlikely (4) to Extremely Unlikely (7). On average, for the Perceived Ease of Use scale the process designer responded with 2.88, which approximately relates to Slightly Likely (3). For the Perceived Usefulness scale, in turn, the process designer in average responded with 3.49 , which approximately relates to Slightly Likely (3). Hence, we conclude that process designers find it in average slightly likely that it would be easy to learn and use change patterns.

\subsection{Qualitative Feedback Regarding Change Pattern Usage}

We additionally asked participants for qualitative feedback with the usage of change patterns. The obtained feedback revealed usability issues, which are in line with the results reported in Sect. 4.2 and 4.3, and which at least partially explain why perceived ease of use and perceived usefulness did not receive better scores. The qualitative evaluation shows that perceived ease of use and perceived usefulness of the patterns heavily depends on process characteristics. Several designers stated that they perceive change patterns especially useful for models that are rather simple, since in this case change patterns allow them to speed up modeling. For more complex models (e.g., highly nested models), process designers rather prefer using change primitives, since this gives them more flexibility: "I like both ways of working, with patterns and without. After getting a bit used to the patterns I find them easier to not let me make mistakes, for thinking and sketching I find the other approach easier." Since the available pattern set was limited, process designers were partially forced to delete big fragments when their problem solving strategy turned out to be ineffective (cf. Sect. 4.2 and 4.3). This was especially true for model regions with complex structure requiring more sophisticated problem solving strategies on how to combine patterns. This is reflected by the following statement of one participant: "From my point of view, it always depends on whether the process is clear, which makes it easy to use change patterns. However, if the process somehow is really complex and it's hard to think about everything before starting to model a process it's better to avoid using change patterns, since once you've model something wrong, it could happen that you have to remodel many parts of the process." This statement indicates that limitations of working memory might be a bottleneck, especially when designers lack schemata for efficiently extracting and processing information and problem solving strategies on how to best combine patterns. To combine the strength of patterns-based modeling and the modeling of change primitives, designers expressed the wish for a modeling environment allowing for the combination of both modeling styles: "If the change pattern functionality would be included into standard modeling tools it would be a very useful addition".

\section{Discussion and Limitations}

This section discusses the results and presents limitations that pose potential threats to their validity. 


\subsection{Discussion}

The results described in Sect. 4 reveal that simple control flow structures without any nesting can be well managed by most designers. Probably, the existing schemata for change primitives was useful in simple models. In general, only few problems, which can be directly attributed to pattern usage, could be observed for model parts without any nesting. Even when faced with the modeling environment for the first time, subjects did not have any notable problems when inserting activities in sequences, making an activity optional, or inserting an activity in parallel. There was only one exception where detours in the modeling process were caused by a poor implementation of the graphical layout of the CEP modeler, which will be addressed in a future version of this software to improve perceived ease of use. Faced with more complex control flow structures, in turn, the structural restrictions imposed by modeling based on change patterns led to considerable problems with model construction partially resulting into long detours or incorrect models. These findings are underlined by feedback of the participants who appreciate the correctness-by-construction guarantees, but feel restricted when faced with complex control flow constructs (cf. Sect. 5). Clearly, existing schemata was not helping in the modeling process of such complex structures, forcing designers to create a new plan schema.

Difficulties faced by process designers can partially be explained by the available patterns set. Even though the patterns available to process designers cover all basic control-flow patterns (i.e., sequence, exclusive/parallel branchings, and loops), the pattern set we used turned out to be insufficient for efficient model construction. Especially in the context of Task A most process designers had to delete parts of their model due to dead ends when trying to construct region R16. Having a pattern Move Process Fragment [4] available would presumably address many of the challenges faced by process designers and facilitate resolution of dead ends. Therefore, we plan to extend CEP and to conduct another study to test whether this will lead to the expected benefits and improve perceived ease of use and usefulness of change patterns modeling.

\subsection{Limitations}

As every research, this work is subject to limitations. The fact that the sample size (16 participants) was relatively small certainly constitutes a threat regarding the generalization of our results. In addition, using students instead of professionals poses another validity threat. However, we are mildly optimistic about the usefulness of the presented insights on the basis of modeling behavior of graduate students, since [30] identified that such subjects perform equally well in process modeling tasks as some professional designers. However, we acknowledge that process designers experienced with the usage of change patterns will presumably face less problems during model creation. Another limitation relates to the fact that we used only two different modeling tasks (with different complexity) in our study. The analysis indicated that difficulties during model creation strongly depend on model characteristics. It is questionable in how far 
results may be generalized to models with different characteristics. As a consequence, we plan additional experiments testing the influence of model structure on difficulties in change pattern usage. For some of the deviations in the context of Task B we cannot conclude with certainty from our data whether the problems were caused by pattern usage or insufficient domain knowledge. To single out these factors we will conduct further studies with a setup as suggested in [8]. Regarding the internal validity, to alleviate the thread related to the classification of reasons for deviations, a consensus-building process [29] was performed by two authors of the paper.

\section{Related Work}

Our work is closely related to research on the PPM and process model creation patterns. Research on the process of modeling typically deals with interaction of different parties focusing on structured discussions among system analysts and domain experts [31, 21]. The procedure of developing process models in a team is analyzed in [32] and characterized as negotiation process. Participative modeling is discussed in [33]. Each of these works builds on observations of modeling practice and distills normative procedures for steering the process of modeling toward successful completion. Hereby, the focus is on the effective interaction between the involved stakeholders. Our work is complimentary to this perspective through its focus on the formalization of the process model. The interactions with the modeling environment have been investigated in [11], identifying three distinct modeling styles. In turn, [10] demonstrates that a structured modeling style leads to models of better quality, and $[11,34]$ suggest different visualization techniques for obtaining an overview of the PPM. [35] investigates the PPM using eye movement analysis. Common to all these works is the focus on interactions with the modeling environment using change primitives, while this paper investigates the use of change patterns. Also related to our work is the usage of change patterns for process schema creation. For example, AristaFlow allows modeling a sound process schema based on an extensible set of change patterns [23]. In turn, [36] describes a set of pattern compounds, similar to change patterns, allowing for the context-sensitive selection and composition of workflow patterns during process modeling. Complementary to existing research on process model creation based on patterns, which has a strong design focus, this paper provides first empirical insights into the usage of change patterns.

\section{Summary}

While work related to the PPM has emerged as a new stream of research in recent years, little is known about this process when utilizing change patterns. In this exploratory study we investigate the challenges, process designers are facing when creating process models based on change patterns as well as their subjective perception regarding the usage of these patterns. Our results show that 
process designers face relatively little difficulties when creating simple controlflow structures. When faced with more complex process structures, the structural restrictions imposed by change patterns caused considerable problems for most of the process designers. Building respective structures efficiently (i.e., without detours) requires process designers to look ahead, since patterns cannot always be arbitrarily combined. This need for looking ahead is a fundamental difference compared to process model creation using change primitives and did not only lead to observable difficulties, but was also perceived as challenging and restrictive by subjects. The exploratory study not only confirmed that the creation of process models using change patterns impacts the PPM, but also gives advice regarding the improvement of the modeling approach based on change patterns. In particular, it showed that a basic set of change pattern as used for the exploratory study is not sufficient for efficient model creation.

\section{References}

1. Becker, J., Rosemann, M., Uthmann, C.: Guidelines of Business Process Modeling. In: Business Process Management, Models, Techniques, and Empirical Studies. Springer-Verlag, London, UK (2000) 30-49

2. Kock, N., Verville, J., Danesh-Pajou, A., DeLuca, D.: Communication flow orientation in business process modeling and its effect on redesign success: Results from a field study. Decision Support Systems 46 (2009) 562-575

3. Mendling, J., Verbeek, H.M.W., van Dongen, B.F., van der Aalst, W.M.P., Neumann, G.: Detection and prediction of errors in EPCs of the SAP reference model. Data and Knowledge Engineering 64 (2008) 312-329

4. Weber, B., Reichert, M., Rinderle, S.: Change Patterns and Change Support Features - Enhancing Flexibility in Process-Aware Information Systems. Data and Knowledge Engineering 66 (2008) 438-466

5. Rinderle-Ma, S., Reichert, M., Weber, B.: On the formal semantics of change patterns in process-aware information systems. In: Proc. ER '08. (2008) 279-293

6. Reichert, M., Dadam, P.: ADEPTflex: Supporting Dynamic Changes of Workflow without Losing Control. JIIS 10 (1998) 93-129

7. Casati, F.: Models, Semantics, and Formal Methods for the design of Workflows and their Exceptions. PhD thesis, Milano (1998)

8. Soffer, P., Kaner, M., Wand, Y.: Towards Understanding the Process of Process Modeling: Theoretical and Empirical Considerations. In: Proc. ER-BPM '11. (2012) 357-369

9. Pinggera, J., Zugal, S., Weidlich, M., Fahland, D., Weber, B., Mendling, J., Reijers, H.: Tracing the Process of Process Modeling with Modeling Phase Diagrams. In: Proc. ER-BPM '11. (2012) 370-382

10. Claes, J., Vanderfeesten, I., Reijers, H., Pinggera, J., Weidlich, M., Zugal, S., Fahland, D., Weber, B., Mendling, J., Poels, G.: Tying Process Model Quality to the Modeling Process: The Impact of Structuring, Movement, and Speed. In: Proc. BPM '12. (2012) 33-48

11. Pinggera, J., Soffer, P., Zugal, S., Weber, B., Weidlich, M., Fahland, D., Reijers, H., Mendling, J.: Modeling Styles in Business Process Modeling. In: Proc. BPMDS '12. (2012) 151-166 
12. Gray, P.: Psychology. Worth Publishers (2007)

13. Tracz, W.: Computer programming and the human thought process. Software: Practice and Experience 9 (1979) 127-137

14. Jeffries, R., Turner, A., Polson, P., Atwood, M.: The Process Involved in Designing Software. In: Cognitive Skills and Their Acquisition. Erlbaum (1981) 255-283

15. Rist, R.: Schema Creation in Programming. Cognitive Science 13 (1989) 389-414

16. Kant, E., Newell, A.: Problem Solving Techniques for the design of algorithms. Information Processing \& Management 20 (1984) 97-118

17. Anderson, J.: Acquisition of cognitive skill. Psychological Review 89 (1982) 369406

18. Guindon, R., Curtis, B.: Control of cognitive processes during software design: what tools are needed? In: Proc. CHI '88. (1988) 263-268

19. Sweller, J.: Cognitive load during problem solving: Effects on learning. Cognitive Science (1988) 257-285

20. Brooks, R.: Towards a theory of the cognitive processes in computer programming. International Journal of Man-Machine Studies 9 (1977) 737-751

21. Hoppenbrouwers, S., Proper, H., Weide, T.: A fundamental view on the process of conceptual modeling. In: Proc. ER '05. (2005) 128-143

22. Petre, M.: Why Looking Isn't Always Seeing: Readership Skills and Graphical Programming. Commun. ACM (1995) 33-44

23. Dadam, P., Reichert, M.: The ADEPT project: a decade of research and development for robust and flexible process support. Comp Scie - R\&D 23 (2009) 81-97

24. Pinggera, J., Zugal, S., Weber, B.: Investigating the Process of Process Modeling with Cheetah Experimental Platform. In: Proc. ER-POIS '10. (2010) 13-18

25. Davis, F.: Perceived usefulness, perceived ease of use, and user acceptance of information technology. MIS Quarterly 13 (1989) 319-340

26. Pinggera, J., Zugal, S., Weber, B., Fahland, D., Weidlich, M., Mendling, J., Reijers, H.: How the Structuring of Domain Knowledge Can Help Casual Process Modelers. In: Proc. ER '10. (2010) 445-451

27. Fahland, D., Woith, H.: Towards process models for disaster response. In: Proc. PM4HDPS 2008. (2008) 254-265

28. Reichert, M., Weber, B.: Enabling Flexibility in Process-Aware Information Systems: Challenges, Methods, Technologies. Springer (2012)

29. Recker, J., Safrudin, N., Rosemann, M.: How novices design business processes. Inf. Syst. 37 (2012) 557-573

30. Reijers, H., Mendling, J.: A study into the factors that influence the understandability of business process models. IEEE Transactions on Systems Man and Cybernetics, Part A 41 (2011) 449-462

31. Frederiks, P., Weide, T.: Information modeling: The process and the required competencies of its participants. Data and Knowledge Engineering 58 (2006) 4-20

32. Rittgen, P.: Negotiating Models. In: Proc. CAiSE '07. (2007) 561-573

33. Stirna, J., Persson, A., Sandkuhl, K.: Participative Enterprise Modeling: Experiences and Recommendations. In: Proc. CAiSE '07. (2007) 546-560

34. Claes, J., Vanderfeesten, I., Pinggera, J., Reijers, H., Weber, B., Poels, G.: Visualizing the Process of Process Modeling with PPMCharts. In: Proc. TAProViz '12. (2013) 744-755

35. Pinggera, J., Furtner, M., Martini, M., Sachse, P., Reiter, K., Zugal, S., Weber, B.: Investigating the Process of Process Modeling with Eye Movement Analysis. In: Proc. ER-BPM '12. (2013) 438-450

36. Gschwind, T., Koehler, J., Wong, J.: Applying patterns during business process modeling. In: Proc BPM'08. (2008) 4-19 